\title{
A case of pericardial tamponade caused by abdominal knife stab
} injury

\author{
Zhongping Cao* \\ Department of Anesthesia, The Special Characteristic Medicine Center of Chinese People Armed Police Force, Tianjin 300162, China.
}

\author{
*Corresponding Author: Zhongping Cao \\ Chenglin Road, NO 220, Department of Anesthesia, \\ The Special Characteristic Medicine Center of Chinese \\ People Armed Police Force, Tianjin 300162, China. \\ Tel: +862260577658 \\ Fax: +862260577658 \\ Email: 09czp@163.com
}

Received: Dec 05, 2020

Accepted: Dec 21, 2020

Published: Dec 24, 2020

Archived: www.jcimcr.org

Copyright: (C) Cao Z (2020).

\begin{abstract}
This paper reported a case of pericardial tamponade was caused by the blood from abdominal organ injury, which flowed through the diaphragm and pericardial holes into the pericardium, liver injury, gastric injury, pancreatic injury, diaphragmatic break were found by the following laparotomy exploration.
\end{abstract}

Keywords: Pericardial tamponade, Hemorrhagic shock, Abdominal stab injury

\section{Case report}

A male, 41 year old patient, with emergency pericardial tamponade and abdominal knife stab wound was admitted into the operation room for open chest and abdominal exploration. The patient showed symptoms of lip cyanosis, shortness of breath, with a blood pressure of $120 / 85 \mathrm{mmHg}$, heart rate of 132 beats / $\min$, oxygen saturation of $92 \%$. The patient kept a half sitting posture and a condition of full stomach. The patient was performed left radial artery and right internal jugular vein puncture anesthetized with lidocaine. Blood gas analysis showed that, $\mathrm{pH}$ : 7.31, $\mathrm{PaCO}_{2}$ : $37 \mathrm{mmHg}, \mathrm{PaO}_{2}$ : $199 \mathrm{mmHg}$, BE: -7.7, НCT: 34\%. Tracheal intubation was performed with fast induction (midazolam $4 \mathrm{mg}$, cisatracurium $0.2 \mathrm{mg} / \mathrm{Kg}$, sufentanil $40 \mu \mathrm{g}$, propofol 1 $\mathrm{mg} / \mathrm{Kg}$ ) after rapid acidosis correction and volume expansion. Pericardium diaphragmatic surface break and no damage to the heart was observed by median emergency thoracotomy exploration. The pericardial hemorrhage was originated from abdominal organ injury, the chest was closed after cleaning up the pericardial hemorrhage, repairing the pericardium and chest exploration, no other organs in the chest was injuried. Liver injury, gastric injury, pancreatic injury, diaphragmatic break were found by the following laparotomy exploration, then gastric partial resection and repair, liver rupture repair, pancreatic tail repair and diaphragm repair were performed. Pericardial tamponade was caused by the blood from abdominal organ injury, which flowed through the diaphragm and pericardial holes into the pericardium. The operation lasted $5.5 \mathrm{~h}$ with $4000 \mathrm{ml}$ intraoperative bleeding, a total of $10 \mathrm{u}$ red blood cells and $1000 \mathrm{ml}$ fresh frozen plasma were transfused during the operation, the patient returned to the ICU after the operation.

The key to anesthesia management of the patients were the following: firstly, the conventional treatment of full stomach, inhibition of gastric acid secretion, gastrointestinal decompression and anticholinergic treatment were adopted, instead of positive pressure ventilation treatment, preoxygenation treatment and cricoid pressure were adopted during fast induction phase 
Citation: Cao Z. A case of pericardial tamponade caused by abdominal knife stab injury. J Clin Images Med Case Rep. 2020;

1(1): 1001.

to prevent the gastric contents aspiration and break into the abdominal cavity. Tracheal intubation was performed by maintaining $100 \%$ oxygen saturation. Secondly, the internal jugular vein puncture was performed guided by ultrasound at a half sitting position under local anesthesia. Patient was kept in a suitable posture, and the drug, liquid channel and CVP monitoring were ensured. Thirdly, the patient's special position provides the following information: in this case, half sitting position could help to drain the blood in the pericardium to the abdominal cavity and relieve the symptom of pericardial tamponade. The most important was the treatment of hemorrhagic shock. The measures included acidosis correction, volume expansion, red blood cells and fresh frozen plasma transfusion, vital signs monitoring and effective treatment [1].

\section{References}

1. Simon RF, Jean LV. Circulatory Shock. N Engl J Med 2013; 369: 1726-34. 